\title{
Vascular Endothelial Dysfunction and Autonomic Nervous Hyperactivity among Premenopausal Women with Cold- Sensitivity Constitution (Hiesho)
}

\author{
Kaori Kono, ${ }^{1,2}$ Shichiro Abe, ${ }^{1}$ Machiko Yamamoto, ${ }^{3}$ Ryo Kayashima, ${ }^{2}$ \\ Kentaro Kaneko, ${ }^{3}$ Masashi Sakuma, ${ }^{1}$ Shigeru Toyoda, ${ }^{1}$ Toshiaki Nakajimaa ${ }^{1}$ and \\ Teruo Inoue ${ }^{1}$ \\ ${ }^{1}$ Department of Cardiovascular Medicine, Dokkyo Medical University School of Medicine, Shimotsuga-gun, \\ Tochigi, Japan \\ ${ }^{2}$ Department of Fundamental Nursing, Dokkyo Medical University School of Nursing, Shimotsuga-gun, Tochigi, \\ Japan \\ ${ }^{3}$ Ibaraki Christian University Graduate School of Nursing, Hitachi, Ibaraki, Japan
}

\begin{abstract}
The cold-sensitivity constitution (CSC), termed "Hiesho" in Japanese, is a woman-specific cold sense of peripheral sites. The etiology of and criteria for CSC are not yet well established. We defined CSC as temperature gradient $>6^{\circ} \mathrm{C}$ between body surface and core, and investigated the autonomic nervous activity by measuring heart rate variability and the vascular endothelial function by determining reactive hyperemia index $(\mathrm{RHI})$ in 43 healthy premenopausal women, aged 18-47 years. Twenty five women had CSC during both the follicular and luteal phases of their menstrual cycles (sustained-CSC group), 8 women did not show CSC during both phases (non-CSC group), and the remaining 10 women showed CSC in either menstrual phase (occasional CSC). To identify the pathophysiological bases of CSC, we compared the sympathetic nervous activity and vascular endothelial function between sustained-CSC and non-CSC. We thus found that sympathetic nervous activity was higher among the sustained-CSC group $(p=0.042)$ during the follicular phase, compared with the non-CSC group, while the RHI was similar in both groups. Furthermore, the sympathetic nervous activity was similar between the sustained-CSC women aged $\geq 40$ years $(n=10)$ and those aged $<40$ years $(n=15)$ during either menstrual phase, whereas the RHI of the women aged $<40$ years was lower during the follicular phase $(p=0.045)$, compared with the women aged $\geq 40$ years. In conclusion, CSC is associated with sympathetic nervous hyperactivity in premenopausal women, and vascular endothelial dysfunction is also involved in CSC among younger women.
\end{abstract}

Keywords: autonomic nervous activity; cold-sensitivity constitution; heart rate variability; reactive hyperemiaperipheral arterial tonometry; vascular endothelial function

Tohoku J. Exp. Med., 2021 January, 253 (1), 51-60.

\section{Introduction}

A cold-sensitivity constitution (CSC), termed "Hiesho" in Japanese, is a sensation of coldness, in which an individual perceives extremities to be cold or is unable to warm them. Formerly, CSC was seen mainly in menopausal women, whose serum estrogen levels were decreased (Melby 2007). Recently, however, CSC has often been seen even among younger women (Kimura and Asami 2016). The CSC is mainly considered to be an indefinite complaint and not life-threatening, and therefore is not a focus of medical care. Nevertheless, the CSC is often associated with other subjective complaints such as thirst, headache, edema, shoulder stiffness, and fatigue, in addition to a sense of coldness (Mori et al. 2018); all of which substantially leads to a poor quality of life, and possibly leads to another illness, such as depression. Although a number of women are affected by a CSC, the etiology of and criteria for the CSC are not yet well established. In addition, whether the CSC is modulated by the menstrual cycle has

Received October 2, 2020; revised and accepted December 16, 2020. Published online January 16, 2021; doi: 10.1620/tjem.253.51. Correspondence: Shichiro Abe, M.D., Department of Cardiovascular Medicine, Dokkyo Medical University School of Medicine, 880 Kitakobayashi, Mibu-machi, Shimotsuga-gun, Tochigi 321-0293, Japan.

e-mail: abenana@dokkyomed.ac.jp

C2021 Tohoku University Medical Press. This is an open-access article distributed under the terms of the Creative Commons Attribution-NonCommercial-NoDerivatives 4.0 International License (CC-BY-NC-ND 4.0). Anyone may download, reuse, copy, reprint, or distribute the article without modifications or adaptations for non-profit purposes if they cite the original authors and source properly.

https://creativecommons.org/licenses/by-nc-nd/4.0/ 
not been clarified.

Although the CSC depends on an individual's perception, its pathophysiological manifestations are a decrease in skin temperature and a disturbance in the peripheral circulatory system (Takatori 1992; Ushiroyama 2005; Nakamura 2008; Ogata et al. 2017), both of which might be closely associated with hyperactivity of the sympathetic nervous system (Ogata et al. 2017). The peripheral circulation depends upon vascular endothelial function, which is modulated by vascular inflammation and oxidative stress (Inoue and Node 2006).

Reactive hyperemia-peripheral arterial tonometry (RH-PAT) is an established method for estimating the endothelial function of the microvasculature (Liu et al. 2009). Estrogen, the production of which declines after the age of 40 years, affects both vascular endothelial function and the autonomic nervous activity of women (Lenton et al. 1984; Hashimoto et al. 1995; Kawano et al. 1996; Tsutsumi et al. 2003).

To our best knowledge, there are no published reports on both the autonomic nervous activity and vascular endothelial function in women with CSC. Furthermore, objective criteria for the identification of CSC have not yet been established. Hence, in this study of premenopausal women during the follicular phase and luteal phase of their menstrual cycle, we used RH-PAT and electrocardiography to assess microvascular endothelial function and heart rate variability. We also measured biomarkers relevant to vascular inflammation and oxidative stress.

\section{Materials and Methods}

\section{Study participants and protocol}

The study participants included 43 healthy premenopausal women, aged $18-47(27.6 \pm 9.5)$ years, who were students or workers at Dokkyo Medical University. After campus interview, they had assented the objective and significance of this study and volunteered to cooperate in (i.e., : non-random assignment). None of the participants had a history of illness, or were taking medications and/or supplements. The study was carried out from October 2018 to April and from December 2019 to February 2020. Takatori et al. (1991) demonstrated that the difference between the highest temperature of the body and the lowest temperature of the limbs was observed in $88 \%$ of the women complaining coldness when the average atmospheric temperature in a month was more than $15^{\circ} \mathrm{C}$. Therefore, we carried out this study from autumn to winter.

The normal menstrual cycle was defined as ranging from 25 to 38 days (Mihm et al. 2011). Depending on each participant's self-assessment of prior menses, the duration of each menstrual period was calculated from the lengths of her last 2 menstrual cycles. The start of menses was determined to be day 1 , and the following period from day 7 to 12 was regarded as the follicular phase, and the subsequent 7 days until the self-predicted next menses as the luteal phase.
Each participant underwent twice tests at both follicular phase and luteal phase, because, in premenopausal period, woman's body temperature fluctuates during menstrual cycle. The tests included temperatures of the right hallux, dorsal aspect of the hand and feet, mesosternum and eardrum; electrocardiography for heart rate variability; RH-PAT for vascular endothelial function; and blood tests. The follicular and luteal phases were distinguished by a difference of $>0.4^{\circ} \mathrm{C}$ between the temperatures of the eardrum at the 2 phases (Stephenson and Kolka 1985). We used a questionnaire to collect information on each participant's age, exercise habit, current smoking, current drinking, and dates of the last 2 menses.

The tests were performed at 7 to $9 \mathrm{AM}$ in a room with controlled temperature and relative humidity, with mean values of $20.3^{\circ} \mathrm{C}$ and $27.2 \%$, respectively. Each participant underwent testing after overnight fasting and abstained from alcohol, smoking, caffeine, and excessive exercise for at least $12 \mathrm{hr}$ before testing. Each participant was advised to obtain sufficient sleep.

This study was conducted in accordance with the Declaration of Helsinki. The study protocol was approved by the local institutional review board (Dokkyo Medical University Review Board: Authorization No. 29014), and written informed consent was obtained from each participant before the study.

\section{Measurement of body temperature}

The eardrum temperature was used for the core temperature. It was measured in the right ear 1 time by a CTD505 ear thermometer (Citizen Systems, Tokyo, Japan). The skin temperatures of the right hallux, dorsal aspect of the hand and feet, and mesosternum were measured simultaneously by a high-accuracy 8-channel wireless thermometer (Nikki-Thermo, Tokyo Japan). The CSC was defined based on prior studies (Takatori 1992; Ogata et al. 2017), as a temperature of the right eardrum minus that of the right hallux equal to $>6^{\circ} \mathrm{C}$.

\section{Reactive hyperemia index}

The RH-PAT device (Endo-PAT2000; Itamar Medical, Caesarea, Israel) was used to determine the reactive hyperemia index (RHI), which reflects microvascular endothelial function (Bonetti et al. 2004; Rubinshtein et al. 2010). The device was placed on the index finger of each hand. A tourniquet cuff was placed around the nondominant forearm. After 5 minutes of rest, the cuff was inflated to $200 \mathrm{mmHg}$, and the pressure was maintained for 5 minutes to induce reactive hyperemia following reperfusion by the cuff deflation. The pulse amplitude, as detected by this device was electronically recorded. The extent of reactive hyperemia was calculated as the ratio of the average pulse amplitude of the signal from the device over an interval of $1 \mathrm{~min}$, starting $1.5 \mathrm{~min}$ after cuff deflation, to the mean pulse amplitude over a 2.5 -min time period before cuff inflation. The RHI value was calculated as the ratio of the reactive 
hyperemia value measured for each hand of the participant. Moreover, the augmentation index (AI) value, which we used to represent the degree of vessel stiffness, and the AI value corrected for a heart rate of $75 \mathrm{bpm}$ were simultaneously calculated. According to the prior study (Tanaka et al. 2018), RHI cutoff values were provided as 1.67 and 2.10 $(<1.67$ for abnormal, $\geq 1.67$ and $<2.10$ for borderline, and $\geq 2.10$ for normal).

\section{Heart rate variability}

After each participant rested in the supine position for 20 min, wireless radiofrequency and electrocardiography sensors (GMS, Tokyo, Japan) were attached to the participant's chest, and electrocardiographic complexes were recorded for 15 minutes. The maximum entropy method and MemCalc/Bonaly Light real-time analysis software (GMS) were used for heart rate variability, which was based on R-R intervals. In the frequency analysis, the ratio of low-frequency (LF) domain $(0.04-0.15 \mathrm{~Hz})$ to high-frequency (HF) domain $(0.15-0.40 \mathrm{~Hz})$ was calculated as mean/10 seconds. The HF domain is generally considered a true indicator of vagal nerve activity. The LF domain reflects the activities of both the vagal and sympathetic nervous systems; thus, the LF/HF ratio is generally considered to be an indicator of sympathetic nervous activity (Kleiger et al. 2005). The reference value of HF is $975 \pm 203 \mathrm{msec}^{2}$ and that of LF/HF ranges from 1.5 to 2.0 (Task Force of the European Society of Cardiology and the North American Society of Pacing and Electrophysiolog 1996).

\section{Blood testing}

Routine tests consisted of the following: blood cell count; fasting glucose; and serum total cholesterol, highdensity lipoprotein cholesterol, low-density lipoprotein cholesterol, and triglycerides (LSI Medience, Tokyo, Japan). High-sensitivity C-reactive protein (hsCRP) was measured by particle-enhanced technology on the Behring BN II nephelometer (Dade Behring, Newark, DE, USA), which used monoclonal anti-CRP antibodies and a calibrator that was traceable to WHO Reference Material (Rifai et al. 1999). Inflammatory biomarkers such as interleukin (IL)-6 and IL-8, and endothelium-related biomarkers such as vascular endothelial growth factor (VEGF) and intercellular molecule-1 (ICAM-1) were measured by the Luminex micro-beads array system (Luminex, Austin, TX, USA), which is a multiplex assay capable of simultaneously quantifying the levels of many biomarkers. The assay was conducted according to the manufacturer's instructions, and used a commercially available kit (BioSource International, Inc., Camarillo, CA, USA) (Carson and Vignali 1999). Assays (Diacron International, Grosseto, Italy) for the derivatives of reactive organ metabolites (d-ROMs) and biological anti-oxidant potential (BAP) were performed to assess oxidative stress status and antioxidant status, respectively (Cornelli et al. 2001).

\section{Statistical analysis}

Data are expressed as means \pm standard deviation for continuous variables and numbers and percentages for categorical variables. The Kolmogorov-Smirnov test with Lilliefors' correlation was used to assess the normality of the distribution of continuous variables. Intergroup comparisons were performed by the unpaired $t$-test for parametric data and the Mann-Whitney $U$-test for nonparametric data. The chi-squared test was used to compare categorical variables. The VEGF data were analyzed as categorical variables, where a value $<$ the limit of measurement sensitivity was considered "negative" and a value $>$ the limit was considered "positive". p values $<0.05$ were considered statistically significant. The Statistical Package for the Social Sciences software (19.0J for Windows, IBM/SPSS, Chicago, IL, USA) was used for analysis.

\section{Results}

\section{Categorization of CSC}

The participants were divided into 3 categories based on time of occurrence of CSC, as follows: (1) CSC during both the follicular phase and luteal phase (sustained CSC), (2) CSC during either the follicular phase or the luteal phase (occasional CSC), and (3) CSC during neither the follicular phase nor the luteal phase (non-CSC). Of 43 participants, sustained CSC was noted in $25(58.1 \%$, aged 30.2 $\pm 10.4)$, non-CSC in $8(18.6 \%$, aged $22.5 \pm 2.9$ years $)$, and occasional CSC in 10 (23.3\%, aged $25.1 \pm 8.5$ years $)$. Of 10 participants categorized with occasional CSC, $8(80 \%)$ manifested CSC during the luteal phase only, and $2(20 \%)$ during the follicular phase only.

Baseline characteristics of participants with sustained CSC vs. non-CSC

To identify significant characteristics of patients with CSC, we compared participants with sustained CSC ( $\mathrm{n}=$ $25)$ vs. non-CSC $(n=8)$. There were no significant differences in the height, body weight, body mass index, current smoking, drinking, and exercise habits between participants with sustained CSC and those with non-CSC (Table 1).

Variables of participants with sustained CSC vs. non-CSC during each menstrual phase

During both the follicular and luteal phases, temperatures of the right hallux $(p<0.001, p<0.001$; follicular phase and luteal phase, respectively), dorsal aspect of the right hand $(p=0.013, p<0.001)$, and top of the right foot ( $p$ $<0.001, \mathrm{p}<0.001$ ) were lower in participants with sustained CSC compared to those with non-CSC, although the temperatures of the mesosternum and the eardrum were similar between the 2 groups. The heart rate, blood pressure, AI, and AI corrected for $75 \mathrm{bpm}$ were also similar between the 2 groups during each menstrual phase (Table 2). 
Table 1. Comparison of baseline characteristics between sustained-CSC and non-CSC participants.

\begin{tabular}{lcccc}
\hline & $\begin{array}{c}\text { Overall } \\
(\mathrm{n}=43)\end{array}$ & $\begin{array}{c}\text { Sustained CSC } \\
(\mathrm{n}=25)\end{array}$ & $\begin{array}{c}\text { Non-CSC } \\
(\mathrm{n}=8)\end{array}$ & p value \\
\hline Age; $\mathrm{yr}$ & $27.6 \pm 9.5$ & $30.2 \pm 10.4$ & $22.5 \pm 2.9$ & 0.245 \\
Height; cm & $160.2 \pm 5.8$ & $160.2 \pm 6.4$ & $159.7 \pm 5.6$ & 0.845 \\
Weight; $\mathrm{kg}$ & $54.7 \pm 7.7$ & $52.6 \pm 7.2$ & $57.0 \pm 8.1$ & 0.176 \\
BMI; kg/m & $21.3 \pm 2.9$ & $20.5 \pm 2.6$ & $22.3 \pm 2.1$ & 0.085 \\
Current smoking; $\mathrm{n}(\%)$ & $1(2.3)$ & $1(4.0)$ & $0(0)$ & 0.758 \\
Drinking; $\mathrm{n}(\%)$ & $5(11.6)$ & $2(8.0)$ & $1(12.5)$ & 0.578 \\
Exercise habit; $\mathrm{n}(\%)$ & $6(14.0)$ & $6(24.0)$ & $0(0)$ & 0.160 \\
\hline
\end{tabular}

CSC, cold-sensitivity constitution; BMI, body mass index.

Table 2. Comparison of quantitative variables between sustained-CSC and non-CSC participants during each menstrual phase.

\begin{tabular}{|c|c|c|c|c|c|c|}
\hline & \multicolumn{3}{|c|}{ Follicular phase } & \multicolumn{3}{|c|}{ Luteal phase } \\
\hline & $\begin{array}{l}\text { Sustained CSC } \\
\quad(\mathrm{n}=25)\end{array}$ & $\begin{array}{l}\text { Non-CSC } \\
(\mathrm{n}=8)\end{array}$ & $\mathrm{p}$ value & $\begin{array}{l}\text { Sustained CSC } \\
\quad(\mathrm{n}=25)\end{array}$ & $\begin{array}{c}\text { Non-CSC } \\
(\mathrm{n}=8)\end{array}$ & $\mathrm{p}$ value \\
\hline Temperature hallux $\left({ }^{\circ} \mathrm{C}\right)$ & $25.4 \pm 2.3$ & $33.5 \pm 1.2$ & $<0.001$ & $25.1 \pm 2.6$ & $32.8 \pm 1.3$ & $<0.001$ \\
\hline Temperature dorsal hand $\left({ }^{\circ} \mathrm{C}\right)$ & $33.2 \pm 1.5$ & $34.6 \pm 0.5$ & 0.013 & $32.4 \pm 1.8$ & $34.8 \pm 0.4$ & $<0.001$ \\
\hline Temperature dorsal foot $\left({ }^{\circ} \mathrm{C}\right)$ & $29.9 \pm 1.6$ & $32.9 \pm 1.0$ & $<0.001$ & $30.1 \pm 1.8$ & $32.9 \pm 0.9$ & $<0.001$ \\
\hline Temperature mesosternum $\left({ }^{\circ} \mathrm{C}\right)$ & $34.6 \pm 0.4$ & $34.6 \pm 0.6$ & 0.940 & $35.1 \pm 0.6$ & $34.7 \pm 0.6$ & 0.122 \\
\hline Temperature eardrum $\left({ }^{\circ} \mathrm{C}\right)$ & $36.2 \pm 0.4$ & $36.1 \pm 0.4$ & 0.832 & $36.8 \pm 0.4$ & $36.5 \pm 0.7$ & 0.116 \\
\hline Heart rate (bpm) & $60.5 \pm 6.6$ & $61.4 \pm 8.5$ & 0.769 & $61.4 \pm 10.7$ & $60.3 \pm 5.8$ & 0.774 \\
\hline Systolic blood pressure (mmHg) & $102 \pm 9$ & $99 \pm 9$ & 0.444 & $101 \pm 9$ & $104 \pm 10$ & 0.471 \\
\hline Diastolic blood pressure (mmHg) & $64 \pm 8$ & $64 \pm 7$ & 0.807 & $63 \pm 7$ & $63 \pm 8$ & 0.951 \\
\hline $\mathrm{AI}(\%)$ & $1.8 \pm 14.6$ & $-5.8 \pm 8.3$ & 0.173 & $-0.3 \pm 14.4$ & $-6.4 \pm 4.2$ & 0.636 \\
\hline $\mathrm{AI}$ adjusted to $75 \mathrm{bpm}$ & $-6.7 \pm 13.9$ & $-14.6 \pm 6.8$ & 0.135 & $-8.0 \pm 13.3$ & $-12.9 \pm 5.0$ & 0.445 \\
\hline White blood cell $(/ \mu \mathrm{L})$ & $4,836 \pm 1,195$ & $6,450 \pm 2,053$ & 0.065 & $5,348 \pm 1,218$ & $7,312.5 \pm 2,238$ & 0.003 \\
\hline Red blood cell $(/ \mu \mathrm{L})$ & $435 \pm 28$ & $446 \pm 25$ & 0.346 & $442 \pm 29$ & $455 \pm 28$ & 0.280 \\
\hline Hemoglobin (g/dL) & $14.3 \pm 6.1$ & $13.2 \pm 0.7$ & 0.951 & $13.3 \pm 1.1$ & $13.4 \pm 1.0$ & 0.804 \\
\hline Hematocrit (\%) & $40 \pm 4$ & $41 \pm 2$ & 0.885 & $41 \pm 3$ & $42 \pm 3$ & 0.391 \\
\hline Platelet $(/ \mu \mathrm{L})$ & $29.5 \pm 15.1$ & $28.5 \pm 6.5$ & 0.696 & $25.9 \pm 5.4$ & $27.1 \pm 4.0$ & 0.573 \\
\hline Fasting glucose $(\mathrm{mg} / \mathrm{dL})$ & $79.5 \pm 7.3$ & $80.5 \pm 3.6$ & 0.617 & $79.8 \pm 6.6$ & $78.9 \pm 5.5$ & 0.735 \\
\hline Hemoglobin $\mathrm{A} 1_{\mathrm{C}}(\%)$ & $5.2 \pm 0.2$ & $5.1 \pm 0.2$ & 0.408 & $5.2 \pm 0.3$ & $5.1 \pm 0.1$ & 0.334 \\
\hline Triglyceride (mg/dL) & $65 \pm 31$ & $64 \pm 25$ & 0.636 & $58 \pm 24$ & $62 \pm 20$ & 0.654 \\
\hline Total-cholesterol (mg/dL) & $189 \pm 33$ & $174 \pm 22$ & 0.232 & $183 \pm 32$ & $177 \pm 36$ & 0.637 \\
\hline HDL-cholesterol (mg/dL) & $70 \pm 11$ & $64 \pm 9$ & 0.149 & $69 \pm 13$ & $65 \pm 9$ & 0.419 \\
\hline LDL-cholesterol (mg/dL) & $105 \pm 27$ & $96 \pm 21$ & 0.388 & $101 \pm 26$ & $98 \pm 30$ & 0.798 \\
\hline hsCRP (mg/dL) & $0.06 \pm 0.10$ & $0.06 \pm 0.10$ & 0.470 & $0.03 \pm 0.05$ & $0.04 \pm 0.03$ & 0.098 \\
\hline
\end{tabular}

CSC, cold-sensitivity constitution; AI, augmentation index; HDL, high-density lipoprotein; LDL, low-density lipoprotein; hsCRP, high-sensitivity C-reactive protein.

Reactive hyperemia index and heart rate variability in the sustained CSC vs. non-CSC participants

Similar RHI values were found for the sustained CSC vs. non-CSC participants during both the follicular phase $(1.92 \pm 0.60$ vs. $1.65 \pm 0.32)$ and luteal phase $(1.88 \pm 0.41$ vs $1.75 \pm 0.39)($ Fig. 1A). In the sustained CSC participants, the LF/HF ratio was significantly higher during the follicular phase $(1.3 \pm 0.9$ vs. $0.8 \pm 0.3, \mathrm{p}=0.042)$ and tended to be higher during the luteal phase ( $1.2 \pm 1.0$ vs. 0.7 $\pm 0.3, \mathrm{p}=0.053$ ) than in the non-CSC participants (Fig. 1B). In the sustained CSC participants, the HF value tended to be lower during the follicular phase $(644 \pm 451$ vs. $\left.1,757 \pm 1,445 \mathrm{msec}^{2}, \mathrm{p}=0.067\right)$, and was significantly lower during the luteal phase $(549 \pm 453$ vs. $1,187 \pm 791$ $\mathrm{msec}^{2}, \mathrm{p}=0.022$ ) than in the non-CSC participants (Fig. $1 \mathrm{C})$.

Results of blood testing, including biomarker levels, in the sustained CSC vs. non-CSC participants

In the sustained CSC participants, the white blood cell count tended to be lower during the follicular phase $(\mathrm{p}=$ 0.065 ) and was significantly lower during the luteal phase 

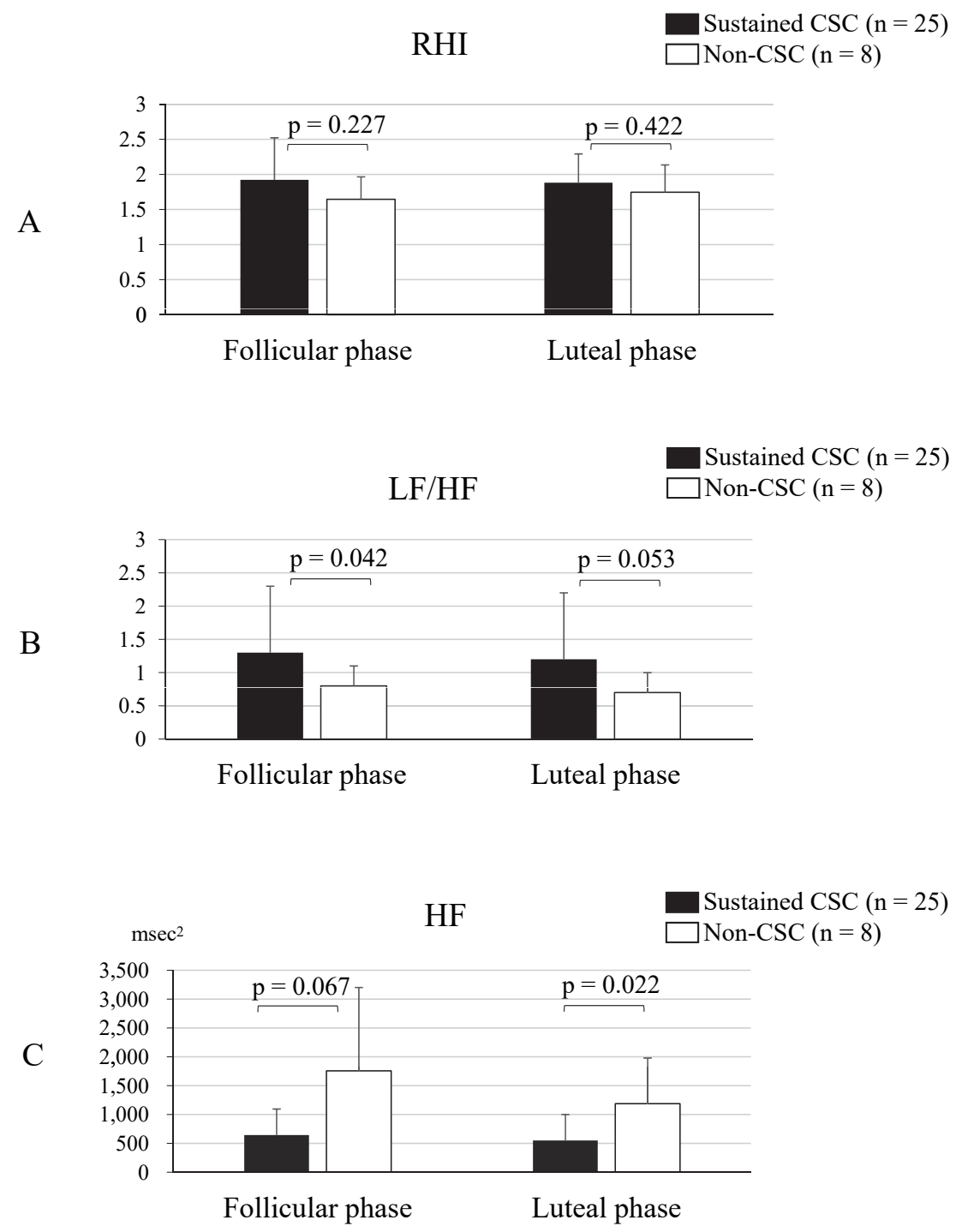

Fig. 1. Comparison between sustained CSC and non-CSC group during menstrual cycle.

RHI, reactive hyperemia index; LF/HF, low frequency/high frequency; HF, high frequency; CSC, cold sensitivity constitution.

than in the non-CSC participants $(\mathrm{p}=0.003)$. The hsCRP level was similar during the follicular phase between both the sustained CSC and non-CSC participants, but the level tended to be lower in the sustained CSC participants during the luteal phase $(p=0.098)$ (Table 2). The level of $d-R O M s$ was similar between the participant groups for each menstrual phase. Although the BAP value during the follicular phase was similar between the sustained CSC and non-CSC participants, the value tended to be higher during the luteal phase in the sustained CSC than in the non-CSC participants $(p=0.098)$. The IL-6, IL- 8 , VGEF, and ICAM- 1 levels were similar between the sustained CSC and non-CSC participants during each menstrual phase (Table 3).

Comparison between the sustained CSC participants aged $<40$ vs. those aged $\geq 40$

As mentioned in the introduction, we divided participants into young and elder women at age of 40 according to prior studies (Lenton et al. 1984; Hashimoto et al. 1995; Kawano et al. 1996; Tsutsumi et al. 2003). Of the 25 participants with sustained CSC, 15 were aged $<40(22.7 \pm$ 4.7) years and 10 were aged $\geq 40(42.0 \pm 2.4$ years: $p<$ $0.001)$. There were no significant differences in the height, body weight, body mass index, current smoking, drinking, and exercise habits between sustained CSC participants aged $<40$ years and those aged $\geq 40$ years (Table 4 ).

In the sustained CSC participants aged $<40$, the RHI value was significantly lower during the follicular phase $(1.73 \pm 0.55$ vs. $2.22 \pm 0.58, p=0.045)$ and tended to be lower during the luteal phase $(1.76 \pm 0.43$ vs. $2.07 \pm 0.33$, p $=0.071)$ than in those aged $\geq 40$ years (Fig. $2 \mathrm{~A}$ ). There was no significant difference in the LH/HF ratio between the sustained CSC participants aged $<40$ years and those aged $\geq 40$ years during both the follicular phase $(1.1 \pm 0.9$ vs. $1.3 \pm 0.7)$ and luteal phase $(1.3 \pm 1.1$ vs. $1.1 \pm 1.0)$ (Fig. 2B). The HF value was significantly higher in the sustained 
Table 3. Comparison of biomarkers relevant to vascular endothelial function between sustained-CSC and non-CSC participants during each menstrual phase.

\begin{tabular}{|c|c|c|c|c|c|c|}
\hline & \multicolumn{3}{|c|}{ Follicular phase } & \multicolumn{3}{|c|}{ Luteal phase } \\
\hline & $\begin{array}{l}\text { Sustained CSC } \\
\quad(\mathrm{n}=25)\end{array}$ & $\begin{array}{l}\text { Non-CSC } \\
\quad(\mathrm{n}=8)\end{array}$ & $\mathrm{p}$ value & $\begin{array}{l}\text { Sustained CSC } \\
\qquad(\mathrm{n}=25)\end{array}$ & $\begin{array}{l}\text { Non-CSC } \\
\quad(\mathrm{n}=8)\end{array}$ & $\mathrm{p}$ value \\
\hline d-ROMs; U.CARR & $312 \pm 69$ & $325 \pm 42$ & 0.496 & $323 \pm 68$ & $354 \pm 73$ & 0.284 \\
\hline $\mathrm{BAP} ; \mu \mathrm{M} / \mathrm{L}$ & $2,800 \pm 488$ & $2,807 \pm 160$ & 0.310 & $3,108 \pm 671$ & $2,844 \pm 556$ & 0.098 \\
\hline IL-6; pg/mL & $0.7 \pm 0.5$ & $0.8 \pm 0.5$ & 0.254 & $0.8 \pm 0.6$ & $0.6 \pm 0.2$ & 0.726 \\
\hline IL-8; pg/mL & $2.8 \pm 2.2$ & $2.7 \pm 0.7$ & 0.374 & $2.5 \pm 1.2$ & $2.1 \pm 1.0$ & 0.371 \\
\hline ICAM-1; ng/mL & $114 \pm 20$ & $103 \pm 14$ & 0.236 & $114 \pm 22$ & $107 \pm 14$ & 0.445 \\
\hline VGEF positive; n (\%) & $14(56.0)$ & $5(62.5)$ & 0.539 & $14(56.0)$ & $6(75.0)$ & 0.299 \\
\hline
\end{tabular}

CSC, cold-sensitivity constitution; d-ROMs, derivative reactive oxygen metabolites; BAP, biological antioxidant potential; IL, interleukin; ICAM, intercellular adhesion molecule; VEGF, vascular endothelial growth factor.

Table 4. Comparison of baseline characteristics between sustained-CSC participants aged $<40$ years and those aged $\geq 40$ years.

\begin{tabular}{lccc}
\hline & $\begin{array}{c}\text { Aged }<40 \\
(\mathrm{n}=15)\end{array}$ & $\begin{array}{c}\text { Aged } \geq 40 \\
(\mathrm{n}=10)\end{array}$ & p value \\
\hline Age; yr & $22.7 \pm 4.7$ & $42.0 \pm 2.4$ & $<0.001$ \\
Height; $(\mathrm{cm})$ & $161.1 \pm 7.4$ & $158.9 \pm 4.5$ & 0.413 \\
Weight; $(\mathrm{kg})$ & $52.5 \pm 7.1$ & $52.6 \pm 7.8$ & 0.966 \\
BMI; $\left(\mathrm{kg} / \mathrm{m}^{2}\right)$ & $20.2 \pm 2.0$ & $20.9 \pm 3.4$ & 0.527 \\
Current smoking; $\mathrm{n}(\%)$ & $1(6.7)$ & $0(0)$ & 0.600 \\
Drinking; $(\%)$ & $1(6.7)$ & $2(20.0)$ & 0.346 \\
Exercise habit; n (\%) & $4(26.7)$ & $2(20.0)$ & 0.545 \\
\hline
\end{tabular}

CSC, cold-sensitivity constitution; BMI, body mass index.

CSC participants aged $<40$ years than in those aged $\geq 40$ years both during the follicular phase $(1,131 \pm 1,214$ vs. $\left.295 \pm 299 \mathrm{msec}^{2}, \mathrm{p}=0.045\right)$ and luteal phase $(929 \pm 760 \mathrm{vs}$. $225 \pm 150 \mathrm{msec}^{2}, \mathrm{p}=0.003$ ) (Fig. 2C).

The level of d-ROMs was similar between the sustained CSC participants aged $<40$ years and those aged $\geq$ 40 years similar during each menstrual phase. Although the BAP value was similar between the sustained CSC participants aged $<40$ years and those aged $\geq 40$ years during the follicular phase, the value was significantly higher in the participants aged $<40$ years than in those aged $\geq 40$ years during the luteal phase $(\mathrm{p}=0.007)$. Although the IL-8 level was similar between the participants aged $<40$ years and those aged $\geq 40$ years during the follicular phase, the level was significantly lower in the participants aged $<40$ years than in those aged $\geq 40$ years during the luteal phase ( $p=$ 0.021). The levels of IL- 6 and ICAM-1 were similar between the participants aged $<40$ years vs. those aged $\geq$ 40 years during both menstrual phases. The VEGF positivity rate was similar between the participants aged $<40$ years and those aged $\geq 40$ years during the follicular phase but tended to be lower in the participants aged $<40$ years than in those aged $\geq 40$ years the during the luteal phase (Table 5).

\section{Discussion}

Although the CSC is common in menopausal women because of a decrease in estrogen production, it may also appear in younger women. Therefore, we targeted premenopausal women of a broad age range in this study, since we hypothesized that the mechanism for the development CSC might be different in young women compared with older women. In most previous reports, the identification of CSC was based on subjective symptoms such as cold sensitivity, with a small amount of information being reported on CSC identified by objective criteria. In this study, regardless of a participant's symptoms, we used an objective criterion, namely, the difference between the temperatures of the surface of peripheral skin and the ear drum, representing the core temperature, to identify the CSC. In addition, we divided participants into 3 categories: CSC during both the follicular phase and luteal phase (sustained CSC), CSC during either the follicular phase or the luteal phase (occasional CSC), and CSC during neither the follicular phase nor the luteal phase (non-CSC). We found that sustained CSC was present in $25(58.1 \%)$ and occasional $\mathrm{CSC}$ in $10(23.3 \%)$ of the 43 healthy premenopausal women we assessed. Thus, in our study, $81.4 \%$ of the participants had a CSC. Previous studies reported that a CSC 
A
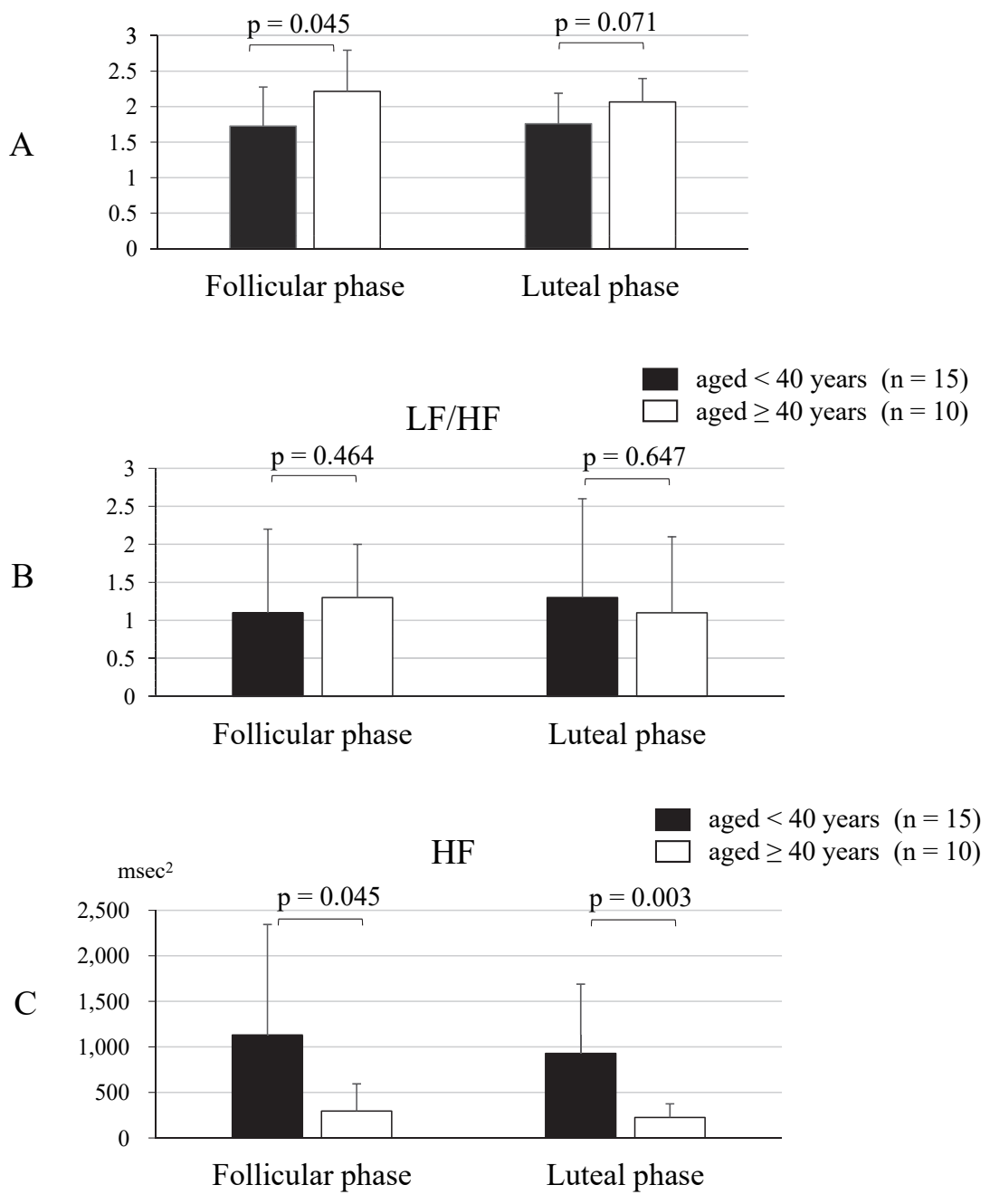

Fig. 2. Comparison between younger and elder women among sustained CSC group.

RHI, reactive hyperemia index; LF/HF, low frequency/high frequency; HF, high frequency.

was present in $50 \%$ of their female participants (Miyamoto et al. 1995; Mori et al. 2018). Thus, we believe that we were able to identify a subclinical CSC by using an objective criterion to identify a CSC.

\section{Occasional CSC}

Because the CSC is usually found in women, it is possibly associated with the menstrual cycle. To our best knowledge, there have been no published reports on the relationship between the menstrual cycle and the incidence of a CSC, especially a CSC identified by a quantitative criterion, as was used in our study. Of the 10 participants identified with occasional CSC, 8 women $(80 \%)$ had CSC during the luteal phase only and $2(20 \%)$ during the follicular phase only. The result suggests that the CSC is easy to be found in the luteal phase, compared with follicular phase. Regardless, when screening for the incidence of CSC, the accurate identification of the menstrual phase of the cycle of each participant is necessary, or to measure body temperatures in both menstrual phases. In this study, to assess the significant characteristics of the CSC, we compared the 2 other groups of participants, those with sustained CSC $(n=25)$ and those with non-CSC $(n=8)$, and excluded the participants with the occasional CSC. Even in our small-sized challenge, we could not conceive exactly how such occasional change associate with mechanism of CSC, and how we should deal with occasional CSC women. Therefore, in this study, we compared the 2 other groups of participants, those with sustained CSC and those with nonCSC, excluding the participants with the occasional CSC, because the sustained CSC might associate to the fundamental mechanism of CSC.

\section{Activity of the autonomic nervous system and CSC}

Changes in the serum levels of estrogen and progesterone during the menstrual cycle affect the mechanisms that 
Table 5. Comparison of biomarkers relevant to vascular endothelial function between sustained-CSC participants aged $<40$ years and those aged $\geq 40$ years.

\begin{tabular}{|c|c|c|c|c|c|c|}
\hline & \multicolumn{3}{|c|}{ Follicular phase } & \multicolumn{3}{|c|}{ Luteal phase } \\
\hline & $\begin{array}{c}<40 y \\
(\mathrm{n}=15)\end{array}$ & $\begin{array}{c}\geq 40 y \\
(\mathrm{n}=10)\end{array}$ & $\mathrm{p}$ value & $\begin{array}{c}<40 \mathrm{y} \\
(\mathrm{n}=15)\end{array}$ & $\begin{array}{c}\geq 40 y \\
(\mathrm{n}=10)\end{array}$ & $\mathrm{p}$ value \\
\hline d-ROMs; U.CARR & $310 \pm 71$ & $315 \pm 70$ & 0.807 & $310 \pm 27$ & $343 \pm 101$ & 0.341 \\
\hline $\mathrm{BAP} ; \mu \mathrm{M} / \mathrm{L}$ & $2,843 \pm 436$ & $2,737 \pm 576$ & 0.338 & $3,364 \pm 744$ & $2,724 \pm 262$ & 0.007 \\
\hline IL-6; pg/mL & $0.8 \pm 0.6$ & $0.6 \pm 0.3$ & 0.892 & $0.8 \pm 0.6$ & $0.8 \pm 0.6$ & 0.935 \\
\hline IL-8; pg/mL & $2.5 \pm 0.9$ & $3.3 \pm 3.4$ & 0.849 & $2.0 \pm 0.8$ & $3.2 \pm 1.3$ & 0.021 \\
\hline ICAM-1; ng/mL & $114 \pm 17$ & $114 \pm 25$ & 0.935 & $117 \pm 11$ & $110 \pm 33$ & 0.495 \\
\hline VGEF positive; n (\%) & $9(60.0)$ & $5(50.0)$ & 0.466 & $6(40.0)$ & $8(80.0)$ & 0.058 \\
\hline
\end{tabular}

CSC, cold-sensitivity constitution; d-ROMs, derivative reactive oxygen metabolites; BAP, biological anti-oxidant potential; IL, interleukin; CAM, intercellular adhesion molecule; VEGF, vascular endothelial growth factor.

establish and regulate the core temperature (Kolka and Stephenson 1997; Inoue et al. 2005; Kuwahara et al. 2005). During the luteal phase, progesterone elevates not only the core temperature's setpoint but also the temperature and blood flow of the forearm (Hessemer and Brück 1985; Stephenson and Kolka 1985; Hassan et al. 1990; Petrofsky et al. 2007). On the other hand, Bartelink et al. (1990) demonstrated that the temperature and blood flow of the fingertip during the luteal phase are lower than those during the follicular phase. They postulated that sympathetic nervous activity and the ratio of estrogen to progesterone might account for the discrepancy.

Sympathetic nervous hyperactivity and vagal hypoactivity appear to be more common during the luteal phase than in the follicular phase (Tada et al. 2017). During the luteal phase, peripheral vasoconstriction via sympathetic nervous hyperactivity may lead to an elevated core temperature. In our results, vagal nervous activity, as demonstrated by HF, tended to be lower during the luteal phase, compared to the follicular phase in all the participants, although sympathetic nervous activity, as demonstrated by LF/HF, was similar between the 2 phases.

We found that sympathetic nervous activity was significantly higher during the follicular phase and tended to be higher during the luteal phase in the sustained CSC participants, compared to the non-CSC participants, while the vagal nervous activity of the sustained CSC participants tended to be lower during the follicular phase and was significantly lower during the luteal phase than those of the non-CSC participants. Contrary to the core temperature, the surface temperature of the extremities was significantly lower in the sustained CSC participants. These results suggest that sympathetic nervous hyperactivity might affect the peripheral circulation, leading to a subsequent decrease in the surface temperature of the extremities, and thus to the incidence of CSC.

We also compared the autonomic nervous activity of the participants $(n=15)$ aged $<40$ years with sustained CSC and that of the participants $(n=10)$ aged $\geq 40$ years with sustained CSC. The sympathetic nervous activity was similar between the 2 groups during both follicular and luteal phases, while the vagal nervous activity was higher in the participants aged $<40$ years during both menstrual phases. The results suggest that sympathetic nervous activity might be independent of aging but vagal nervous activity might decrease with aging, which is consistent with a previous study (Yukishita et al. 2010), also of CSC participants.

\section{Vascular endothelial function and biomarkers}

We assessed the vascular endothelial function of the microvasculature of our study participants using the established method of RH-PAT. Although vascular endothelial function is thought to be associated with sympathetic nervous activity, the RHI, as determined from the RH-PAT method, has not been found to be affected by autonomic nervous activity (Bonetti et al. 2004; Rubinshtein et al. 2010). We found that the RHI was similar in the participants with sustained CSC and those with non-CSC during both menstrual phases. However, the RHI was significantly lower during the follicular phase and tended to be lower during the luteal phase in the sustained CSC participants aged $<40$ years, compared to the sustained-CSC participants aged $\geq 40$ years. This result suggests that impaired microvascular endothelial function might be involved in microcirculatory disturbance, independently of autonomic nervous activity, resulting in the occurrence of a CSC in young women. In women with advanced reproductive age, such as those aged $\geq 40$ years, the follicular fluid contains estrogen, which has a secondary vasodilatory effect via endothelial NO production (Hayashi et al. 1995), which is stronger than the vasodilatory effect occurring in younger women (Klein et al. 1996). Thus, vascular endothelial function might have been maintained in our participants aged $\geq 40$ years but might have been impaired in our participants aged $<40$ years.

We found that the white blood cell count tended to be lower during the follicular phase and was significantly lower during the luteal phase in the participants with sustained CSC, compared to the non-CSC participants. In 
addition, the hsCRP was similar between the 2 groups of participants during the follicular phase and tended to be lower in the sustained CSC participants during the luteal phase, although the mechanism for this finding has not been clarified and we cannot account for it. Although BAP tended to be higher in the sustained CSC participants than in the non-CSC participants during the luteal phase, the other biomarkers for inflammation, endothelial function and oxidative stress status were similar between the 2 groups of sustained CSC and non-CSC participants. Altogether, our findings suggest that the occurrence of the CSC might be independent of vascular endothelial function, inflammatory status and oxidative stress. On the other hand, the BAP level was higher in the sustained CSC participants aged < 40 years during the luteal phase, compared to those aged $\geq$ 40 years. The result suggests that antioxidant potential might decrease in women of advanced reproductive age, compared to that in younger women. Finally, the IL-8 level and the prevalence of VEGF positivity were lower in the sustained-CSC participants aged < years 40 during the luteal phase, compared to those aged $\geq 40$ years during the luteal phase. IL-8 is considered to be an inflammatory cytokine and is simultaneously involved in angiogenesis. Therefore, IL-8 possibly ameliorates vascular endothelial function via the activation of the VEGF receptor and VEGF expression ( $\mathrm{Li}$ et al. 2005; Martin et al. 2009). In our results, the lower IL-8 level and lower prevalence of VEGF positivity might be consistent with reduced microvascular endothelial function in sustained CSC participants aged < 40 years.

\section{Objective criteria for CSC}

In general, CSC is an individual's perception such as cold sensation. When discussing pathophysiology of the CSC, however, its objective criteria would be required. In our study, the CSC was defined based on prior studies (Takatori 1992; Ogata et al. 2017), as a temperature of the right eardrum minus that of the right hallux equal to $>6^{\circ} \mathrm{C}$. We believe this definition would be rationale in advancing this study, in which we divided participants into 3 categories based on occurrence of CSC in each menstrual phase. However, there is no established definition of CSC. From this study, we could suggest that the objective criteria to identify the CSC should be based on difference of body temperature between surface and core in both menstrual phases, in addition to subjective cold sensation. Therefore, a quantitative evaluation of the surface and core temperatures in each menstrual phase would be required for precise diagnosis of the CSC. In addition, evaluation of autonomic nervous activity and vascular endothelial function would be recommended to assess pathophysiology of the CSC.

\section{Study limitations}

This study has several limitations. First, this was a non-randomized single-center study with a small sample size without its determination. Second, the study has a bias for age in the selection of participants, and the results cannot be generalized. Therefore, we need to expand the size of the study. Finally, the absence of the quantification of sex hormones during each menstrual phase is a major limitation. Because serum hormone levels were not measured during each phase of the menstrual cycle, we can only speculate on their interactions.

\section{Conclusion}

A quantitative evaluation of surface and core body temperatures in each menstrual phase may be required for diagnosis of CSC. The CSC might be modulated by the menstrual cycle and might be associated with sympathetic nervous hyperactivity. In younger women, endothelial dysfunction, might be involved in the occurrence of a CSC, independently of sympathetic nervous activity. Evaluation of autonomic nervous activity and vascular endothelial function would be useful to assess pathophysiology of the CSC.

\section{Acknowledgments}

This study was conducted with the support of the Dokkyo Medical University Young Investigator Award (No. 2018-10) and with a grant from the Public Trust Cardiovascular Research Fund. We greatly appreciate the cooperation of all of the study participants, i.e., students and workers at Dokkyo Medical University.

\section{Conflict of Interest}

The authors declare no conflict of interest.

\section{References}

Bartelink, M.L., Wollersheim, H., Theeuwes, A., van Duren, D. \& Thien, T. (1990) Changes in skin blood flow during the menstrual cycle: the influence of the menstrual cycle on the peripheral circulation in healthy female volunteers. Clin. Sci. (Lond.), 78, 527-532.

Bonetti, P.O., Pumper, G.M., Higano, S.T., Holmes, D.R. Jr., Kuvin, J.T. \& Lerman, A. (2004) Noninvasive identification of patients with early coronary atherosclerosis by assessment of digital reactive hyperemia. J. Am. Coll. Cardiol., 44, 21372141.

Carson, R.T. \& Vignali, D.A. (1999) Simultaneous quantitation of 15 cytokines using a multiplexed flow cytometric assay. $J$. Immunol. Methods, 227, 41-52.

Cornelli, U., Terranova, R., Luca, S., Cornelli, M. \& Alberti, A. (2001) Bioavailability and antioxidant activity of some food supplements in men and women using the D-Roms test as a marker of oxidative stress. J. Nutr., 131, 3208-3211.

Hashimoto, M., Akishita, M., Eto, M., Ishikawa, M., Kozaki, K., Toba, K., Sagara, Y., Taketani, Y., Orimo, H. \& Ouchi, Y. (1995) Modulation of endothelium-dependent flow-mediated dilatation of the brachial artery by sex and menstrual cycle. Circulation, 92, 3431-3435.

Hassan, A.A., Carter, G. \& Tooke, J.E. (1990) Postural vasoconstriction in women during the normal menstrual cycle. Clin. Sci. (Lond.), 78, 39-47.

Hayashi, T., Yamada, K., Esaki, T., Kuzuya, M., Satake, S., Ishikawa, T., Hidaka, H. \& Iguchi, A. (1995) Estrogen increases endothelial nitric oxide by a receptor-mediated system. Biochem. Biophys. Res. Commun., 214, 847-855. 
Hessemer, V. \& Brück, K. (1985) Influence of menstrual cycle on shivering, skin blood flow, and sweating responses measured at night. J. Appl. Physiol. (1985), 59, 1902-1910.

Inoue, T. \& Node, K. (2006) Vascular failure: a new clinical entity for vascular disease. J. Hypertens., 24, 2121-2130.

Inoue, Y., Tanaka, Y., Omori, K., Kuwahara, T., Ogura, Y. \& Ueda, H. (2005) Sex- and menstrual cycle-related differences in sweating and cutaneous blood flow in response to passive heat exposure. Eur. J. Appl. Physiol., 94, 323-332.

Kawano, H., Motoyama, T., Kugiyama, K., Hirashima, O., Ohgushi, M., Yoshimura, M., Ogawa, H., Okumura, K. \& Yasue, H. (1996) Menstrual cyclic variation of endotheliumdependent vasodilation of the brachial artery: possible role of estrogen and nitric oxide. Proc. Assoc. Am. Physicians, 108, 473-480.

Kimura, T. \& Asami, T. (2016) Discrimination of hie and hiesho using toe blood pressure in young women. J. Integr. Med., 14, 436-446.

Kleiger, R.E., Stein, P.K. \& Bigger, J.T. Jr. (2005) Heart rate variability: measurement and clinical utility. Ann. Noninvasive Electrocardiol., 10, 88-101.

Klein, N.A., Battaglia, D.E., Miller, P.B., Branigan, E.F., Giudice, L.C. \& Soules, M.R. (1996) Ovarian follicular development and the follicular fluid hormones and growth factors in normal women of advanced reproductive age. J. Clin. Endocrinol. Metab., 81, 1946-1951.

Kolka, M.A. \& Stephenson, L.A. (1997) Effect of luteal phase elevation in core temperature on forearm blood flow during exercise. J. Appl. Physiol. (1985), 82, 1079-1083.

Kuwahara, T., Inoue, Y., Abe, M., Sato, Y. \& Kondo, N. (2005) Effects of menstrual cycle and physical training on heat loss responses during dynamic exercise at moderate intensity in a temperate environment. Am. J. Physiol. Regul. Integr. Comp. Physiol., 288, R1347-1353.

Lenton, E.A., Landgren, B.M., Sexton, L. \& Harper, R. (1984) Normal variation in the length of the follicular phase of the menstrual cycle: effect of chronological age. Br. J. Obstet. Gynaecol., 91, 681-684.

Li, A., Varney, M.L., Valasek, J., Godfrey, M., Dave, B.J. \& Singh, R.K. (2005) Autocrine role of interleukin-8 in induction of endothelial cell proliferation, survival, migration and MMP-2 production and angiogenesis. Angiogenesis, 8, 63-71.

Liu, J., Wang, J., Jin, Y., Roethig, H.J. \& Unverdorben, M. (2009) Variability of peripheral arterial tonometry in the measurement of endothelial function in healthy men. Clin. Cardiol., 32, 700-704.

Martin, D., Galisteo, R. \& Gutkind, J.S. (2009) CXCL8/IL8 stimulates vascular endothelial growth factor (VEGF) expression and the autocrine activation of VEGFR2 in endothelial cells by activating NFkappaB through the CBM (Carma3/Bcl10/ Malt1) complex. J. Biol. Chem., 284, 6038-6042.

Melby, M.K. (2007) Chilliness: a vasomotor symptom in Japan. Menopause, 14, 752-759.

Mihm, M., Gangooly, S. \& Muttukrishna, S. (2011) The normal menstrual cycle in women. Anim. Reprod. Sci., 124, 229-236.

Miyamoto, N., Aoki, T., Muto, N., Inaba, R. \& Iwata, H. (1995) Relationship between chilliness of the limbs and daily-life conditions in young females. Nihon Eiseigaku Zasshi, 49, 1004-1012 (in Japanse).

Mori, H., Kuge, H., Sakaguchi, S., Tanaka, T.H. \& Miyazaki, J.
(2018) Determination of symptoms associated with hiesho among young females using hie rating surveys. J. Integr. Med., 16, 34-38.

Nakamura, S. (2008) Sensitivity to cold in pregnant women: characteristics of skin temperature and its relationship to daily life. J. Jpn. Acad. Nurs. Sci., 28, 3-11.

Ogata, Y., Kaneko, K., Goto, K., Kono, K. \& Yamamoto, M. (2017) Physiological mechanism of hiesho: evaluation by cardiovascular and autonomic dynamics. Jpn. J. Nurs. Sci., 15, 227-234.

Petrofsky, J., Al Malty, A. \& Suh, H.J. (2007) Isometric endurance, body and skin temperature and limb and skin blood flow during the menstrual cycle. Med. Sci. Monit., 13, CR111-117.

Rifai, N., Tracy, R.P. \& Ridker, P.M. (1999) Clinical efficacy of an automated high-sensitivity C-reactive protein assay. Clin. Chem., 45, 2136-2141.

Rubinshtein, R., Kuvin, J.T., Soffler, M., Lennon, R.J., Lavi, S., Nelson, R.E., Pumper, G.M., Lerman, L.O. \& Lerman, A. (2010) Assessment of endothelial function by non-invasive peripheral arterial tonometry predicts late cardiovascular adverse events. Eur. Heart J., 31, 1142-1148.

Stephenson, L.A. \& Kolka, M.A. (1985) Menstrual cycle phase and time of day alter reference signal controlling arm blood flow and sweating. Am. J. Physiol., 249, R186-191.

Tada, Y., Yoshizaki, T., Tomata, Y., Yokoyama, Y., Sunami, A., Hida, A. \& Kawano, Y. (2017) The impact of menstrual cycle phases on cardiac autonomic nervous system activity: an observational study considering lifestyle (diet, physical activity, and sleep) among female college students. J. Nutr. Sci. Vitaminol. (Tokyo), 63, 249-255.

Takatori, A., Okuda, H., Sekiba, K. \& Tanizaki, Y. (1991) Thermological study on the coldness women: second report. The relationship between the change of atmospheric temperature and body surface temperature in women with complaints of coldness in limbs. KankyoByotaikenhokoku, 62, 16-22 (in Japanese).

Takatori, A. (1992) Assessment of diagnostic criterion of coldness in women with thermography. Nihon Sanka Fujinka Gakkai Zasshi, 44, 559-565.

Tanaka, A., Tomiyama, H., Maruhashi, T., Matsuzawa, Y., Miyoshi, T., Kabutoya, T., Kario, K., Sugiyama, S., Munakata, M., Ito, H., Ueda, S., Vlachopoulos, C., Higashi, Y., Inoue, T., Node, K., et al. (2018) Physiological diagnostic criteria for vascular failure. Hypertension, 72, 1060-1071.

Task Force of the European Society of Cardiology and the North American Society of Pacing and Electrophysiolog (1996) Heart rate variability: standards of measurement, physiological interpretation and clinical use. Circulation, 93, 1043-1065.

Tsutsumi, Y., Furukawa, K., Kanno, Y., Maruyama, R., Sato, H. \& Yamamoto, M. (2003) Influence of normal menstrual cycle on autonomic nervous activity and QT dispersion. Jpn. J. Electrocardiology, 23, 207-212.

Ushiroyama, N. (2005) Chill sensation: pathological findings and its therapeutic approach. Igakunoayumi, 215, 925-929 (in Japanese).

Yukishita, T., Lee, K., Kim, S., Yumoto, Y., Kobayashi, A., Shirasawa, T. \& Kobayashi, H. (2010) Age and sex-dependent alterations in heart rate variability: profiling the characteristics of men and women in their 30s. Anti-Aging Medicine., 7, 94-99. 